\title{
THIS WEEK
}

EDITORIALS
CARBON End the one-hit wonders of climate-change action $\mathbf{p . 1 3 0}$
WORLD VIEW Structural

changes to tackle sexual

harassment p.131
TRUMPET NOSE Fossil shows

animal had handy

hidden hooter $\mathbf{p} \mathbf{1 3 3}$

\section{Benefits of sharing}

\section{A swift and effective response to emerging infectious diseases demands that researchers have ready access to the latest data on the pathogens responsible. There is still a long way to go to ensure this.}

$\mathrm{A}$ nother year, another virus. As the Ebola-virus epidemic recedes, Zika dominates the news. The virus, which usually causes only mild symptoms, has been linked to a reported increase in the number of babies born in Brazil with microcephaly - abnormally small heads and brains. The possible implications of this for pregnant women demand a rapid and evidence-based approach.

The immediate priorities are to gather epidemiological and clinical data to establish whether the apparent spike in cases is real and, if so, to what extent the Zika virus is involved (see page 142). And researchers elsewhere must have full access to all of this information as soon as it is available.

Conventional scientific publishing, based on rounds of peer review, can be too slow to rapidly disseminate research findings during a public-health emergency. One solution is the immediate release of data to public databases and subsequent publication of peer-reviewed analysis. As we have said before, prior release of data and analysis to public databases, preprint servers and forums will not jeopardize consideration of a submission to Nature journals. And Nature journals will make all papers relating to Zika virus free to access until further notice.

Already, there have been promising moves to make data on microcephaly, and on the epidemic of Zika in the Americas, readily accessible. The World Health Organization (WHO) has announced a 'Zika Open' initiative, in which all relevant submissions to its Bulletin will be posted online within 24 hours.

In this issue, we publish research that demonstrates the need for rapid data sharing during outbreaks. As detailed on page 228, genomesequencing technology has advanced to the point at which the whole genome of a virus sample can be sequenced in the field within 24 hours by means of a portable sequencing system. Previously, such sequencing during the course of an epidemic has been slower because it has relied on sending samples to a laboratory. Although this method still faces technical challenges, it should prove a crucial tool in epidemiological research. It offers the potential to rapidly trace how a disease is being passed from person to person, and so help to guide authorities to direct resources that can break these transmission chains.

But the full potential of this advance can be realized only if scientists can access sequence data obtained from samples taken at different times and places during an outbreak. Pathogen genome sequences are most useful when studied alongside epidemiological and clinical data.

Many scientists across the research fields, but notably in genomics, have been enthusiastic champions of early data release. Infectiousdisease researchers make use of public forums and databases such as virological.org and GISAID (Global Initiative on Sharing All Influenza Data). The former resource is becoming a major platform for sharing and discussing preliminary analyses of data and it already hosts a genomic analysis of the Zika virus. And, launched just over four years ago, ISARIC (International Severe Acute Respiratory and Emerging Infection Consortium) works with clinicians and epidemiologists to put in place pre-agreed protocols and data-sharing processes that can then quickly be adapted to a new situation.

Almost one year ago, this journal published a Comment article that called for the immediate sharing of outbreak data, a policy that the authors themselves had adopted during their early sequencing of Ebola-virus genomes in the 2014-15 outbreak (N. L. Yozwiak et al. Nature 518, 477-479; 2015). They also called on the WHO to convene a meeting to develop guidance for data sharing during infectious-disease outbreaks. Such a meeting took place last September. It was attended by government representatives, public-health agencies, scientists, research funders, ethicists and publishers. All acknowledged that pathogen sequence information collected during a public-health emergency is of greatest value when released openly, in as close to real time as possible.

A statement released by the WHO after the meeting emphasized the "fundamental moral obligation" of every researcher who generates information related to a public-health emergency to share their preliminary results once these have undergone quality control. Representatives from leading biomedical journals also unequivocally emphasized that disclosure of such information would not prejudice journal publication.

Scientists still face challenges to swift data sharing. For example, as was seen during the emergence of the H5N1 and H7N9 avian influenza viruses and Middle East respiratory syndrome coronavirus, rapid data sharing can be hampered by a lack of international rules that govern how credit and rights (including intellectual property) should be fairly distributed among scientists and authorities in the countries where outbreaks occur as well as researchers based elsewhere.

To play our part in driving the shift towards fast data sharing during public-health emergencies, Nature journals will now encourage authors who haven't already deposited their relevant sequence information in public archives to do so on submission. -

\section{A good precedent Jimmy Carter's efforts to eradicate Guinea worm should be applauded.}

W hen former US President Jimmy Carter wanted Ghana to take his goal to eradicate the Guinea worm seriously, he came up with a novel threat. Carter told the country's president that he would try to get the parasitic disease's name changed to Ghana worm. "There isn't a Guinea worm left in Ghana now," Carter told journalists with a grin in London last week.

Carter might just be the United States' most productive ex-president. In the 35 years since he left the White House, the peanut farmer from 